\title{
APLICACIÓN DEL PROCESO DE ANÁLISIS JERÁRQUICO PARA LA EVALUACIÓN DEL RIESGO DE INCENDIO EN LA IN- DUSTRIA COLOMBIANA.
}

\author{
Julián Uribe Botero', Libardo Antonio Londoño Ciro²
}

\begin{abstract}
1 Ingeniero mecánico, Magíster(c) en gestión integral de riesgos laborales. Politécnico Colombiano Jaime Isaza Cadavid. email: juribo8@hotmail.com.

${ }^{2} \mathrm{PhD}$ en Ingeniería, Director de calidad académica de la institución Universitaria Digital de Antioquia.

email: libaranto@gmail.com.
\end{abstract}

\section{RESUMEN}

La gestión de las emergencias hace parte fundamental de la seguridad y salud en el trabajo. Los incendios se constituyen como las emergencias más comunes en las industrias de nuestro país y en todo el mundo. Por medio del proceso de análisis multi atributo, específicamente el proceso de análisis jerárquico (AHP), los factores de riesgo fueron identificados en las investigaciones que se han realizado usando este método de análisis. El nivel de importancia de cada factor fue calculado por medio de un grupo de expertos que aplicaron el proceso de análisis jerárquico. Los resultados de la investigación pueden ser usados para tener una evaluación detallada el riesgo de incendio que permitiría a los inversionistas o propietarios tomar medidas para reducir las posibles pérdidas en caso de incendio; a las compañías de seguro realizar incrementos en las primas de riesgo basados en la evacuación del riesgo; a los departamentos de bomberos el nivel de cumplimiento de los requerimientos normativos.

Palabras clave: factores de riesgo, proceso de análisis jerárquico, riesgo de incendio, nivel de importancia.

Recibido: 15 de enero de 2021. Aceptado: 25 de abril de 2021

Received: January 15, 2021. Accepted: April 25, 2021

DOI: http://dx.doi.org/10.33571/rpolitec.v17n33a5

\section{APPLICATION OF ANALYTIC HIERARCHY PROCESS IN FIRE RISK ASSESSMENT OF INDUSTRIAL OCCUPANCIES}

\begin{abstract}
Emergency management is a fundamental part of occupational safety and health. Fires are the most common emergencies in the industries of our country and throughout the world. Applying the multi-criteria analysis method, the investigations using the analytic hierarchy process (AHP) in fire risk assessment allowed the identification of fire risk factor. An experts group was consulted about the level of importance of fire risk factors in the fire risk evaluation, for these purpose each expert used the AHP. Results of research can be use to perform the detailed assessment of fire risk, allowing state owners and investors to carry out actions for reduce possible losses in case of fire. Insurance companies can perform the detailed assessment of fire risks on the object and to calculate a risk extra charge to an insurance premium. Fire departments can determine the compliance of a condition of object with requirements of regulations.
\end{abstract}

Keywords: risk factors, analytic hierarchy process, fire risk, priority.

Cómo citar este artículo: J.U. Botero, L.A. Londoño-Ciro. "Aplicación del proceso de análisis jerárquico para la evaluación del riesgo de incendio en la industria colombiana”, Revista Politécnica, vol. 17, no.33 pp.64-75, 2021. DOI: http://dx.doi.org/10.33571/rpolitec.v17n33a5 


\section{INTRODUCCIÓN}

Gestionar la seguridad y la salud en los lugares de trabajo contempla la necesidad de gestionar las emergencias que se puedan presentar. Según el decreto 1072 del año 2015, los empleadores deben implementar y mantener las disposiciones necesarias en materia de prevención, preparación y respuesta ante emergencias. Los incendios, no solo en Colombia, sino en el mundo, se constituyen como emergencias comunes. Un incendio mayor en una industria puede ocasionar daños materiales, muertes, interrupción del negocio y pérdidas de mercado; en efecto el $40 \%$ de los pequeños negocios no asegurados no reabren después de un incendio mayor [1]

El proceso de gestión de riesgos propuesto por la ISO 31000, risk management-Principles and guidelines del año 20089, plantea que el tratamiento del riesgo y el establecimiento de controles se debe realizar después de la valoración de los riesgos (risk assessment) con la finalidad de llevar el riesgo al nivel aceptable para la organización. Para el caso de la gestión del riesgo de incendio, la evaluación se ha realizado históricamente de diferentes maneras; en su libro denominado Evaluation of Fire Safety, los autores explican los modelos probabilísticos, deterministas y estocásticos para valorar el riesgo de incendio y encuentran los métodos de ranking, de índex o métodos por puntos muy aplicables y útiles cuando se desea una cuantificación del riesgo de incendio donde no se requiere gran sofisticación y donde la priorización es el objetivo fundamental y un análisis teórico a profundidad no es costo eficiente o apropiado [2].

Los modelos de valoración del riesgo de incendio como el Meseri, Gustav Purt, Max Gretener y DOW (industria química) son sistemas de valoración de seguridad contra incendio multi atributos, sin embargo, ninguno de ellos hace una distinción entre la intensidad y la importancia de los atributos del riesgo. El proceso de análisis jerárquico (AHP) es una herramienta poderosa para la realización de valoraciones multi atributo [2], ha sido ampliamente revisado y aplicado en la literatura, además permite diferencias la intensidad o grado de importancia de los atributos.

\section{PROCESO DE ANALISIS JERARQUICO}

El proceso de análisis jerárquico (AHP) es una metodología de análisis multiatributo o multicriterio desarrollada por el doctor Thomas L. Saaty [3]. Este método fue diseñado para resolver problemas complejos de criterios múltiples. El resultado del AHP es una jerarquización de prioridades que muestran la preferencia global para cada una de las alternativas de selección. El AHP, mediante la construcción de un modelo jerárquico, permite de manera gráfica y eficiente organizar la información de un problema (en el caso de este artículo el riesgo de incendio), descomponerla y analizarla por partes.

El proceso de análisis jerárquico se fundamenta en:

- La estructuración de un modelo jerárquico.

- Priorización de los elementos.

- Comparaciones pareadas entre los elementos.

- Evaluación de los elementos mediante la asignación de pesos.

- Nivel de importancia de las alternativas de acuerdo con los pesos.

- Síntesis.

- Análisis de consistencia.

Las comparaciones pareadas entre los elementos son fundamentales, el AHP utiliza una escala de 1 a 9 para calificar la preferencia o relevancia de los elementos. 
Tabla 1: Escala fundamental para la calificación de preferencias en AHP [4].

\begin{tabular}{|c|c|c|}
\hline $\begin{array}{l}\text { Escala } \\
\text { numér } \\
\text { ica }\end{array}$ & Escala verbal & Explicación \\
\hline 1 & Igual importancia & $\begin{array}{l}\text { Los dos elementos contribuyen } \\
\text { igualmente a la propiedad o } \\
\text { criterio. }\end{array}$ \\
\hline 3 & $\begin{array}{l}\text { Moderadamente más importante } \\
\text { un elemento que el otro }\end{array}$ & $\begin{array}{l}\text { El juicio y la experiencia previa } \\
\text { favorecen a un elemento frente al } \\
\text { otro. }\end{array}$ \\
\hline 5 & $\begin{array}{l}\text { Fuertemente más importante un } \\
\text { elemento que en otro }\end{array}$ & $\begin{array}{l}\text { E1 juicio y la experiencia previa } \\
\text { favorecen fuertemente a un } \\
\text { elemento frente al otro. }\end{array}$ \\
\hline 7 & $\begin{array}{l}\text { Mucho más fuerte la importancia } \\
\text { de un elemento que la del otro, }\end{array}$ & $\begin{array}{l}\text { Un elemento domina fuertemente. } \\
\text { Su dominación está probada en } \\
\text { práctica }\end{array}$ \\
\hline 9 & $\begin{array}{l}\text { Importancia extrema de un } \\
\text { elemento frente al otro. }\end{array}$ & $\begin{array}{l}\text { Un elemento domina al otro con } \\
\text { el mayor orden de magnitud } \\
\text { posible }\end{array}$ \\
\hline
\end{tabular}

Una vez las comparaciones entre atributos han sido realizadas y se tiene el grado de importancia de los atributos es necesario chequear el grado de consistencia obtenido.

La idea de la consistencia es ilustrada en el siguiente ejemplo: En una matriz de comparación, si proporcionamos un valor de 3 al primer criterio sobre el segundo y asignamos un valor de 3 al segundo criterio con respecto al tercero, el valor de preferencia del primer criterio con respecto el tercero debería ser $3 \times 3=9$. Sin embargo, si el tomador de decisiones ha asignado un valor como 5 o 7, habría un cierto nivel de inconsistencia en la matriz de juicios.

La seguridad contra incendios es un problema complejo que involucra o se ve afectado por múltiples factores, por esta razón la generación o selección de atributos es un paso de vital importancia en el desarrollo del modelo Jerárquico. Algunos autores sugieren la revisión de la literatura o el uso de un panel de expertos para identificar los atributos de un problema en particular [4].

La selección de los atributos es usualmente más arbitraria con resultados dispares; por esta razón la selección de los atributos recae sobre las siguientes categorías: Delphi o un proceso de consenso formal de expertos, escenarios de incendio basados en datos estadísticos, Arboles de análisis de procesos jerárquicos [2]

\section{RESULTADOS}

\subsection{Estructuración del modelo jerárquico}

Para la selección de los atributos se realizó una revisión sistemática de estudios que utilizaron el proceso análisis jerárquico en la evaluación del riesgo de incendio; el resultado de esta búsqueda fue complementada con una búsqueda manual.

Los términos empleados para la búsqueda fueron los siguientes: Risk Assessment AND Fire evaluation AND Hierarchy. Para tener unos resultados más precisos se afinaron los parámetros de búsqueda avanzada para que en el titulo aparecieran los siguientes términos: Fire AND Risk Assessment. Las bases de datos consultadas fueron: Sciencedirect, Medlineplus, Web of science y Cochrane.

Después de la revisión de los artículos que se muestran en la tabla 2 se seleccionaron las categorías definidas en el artículo "Applying Multi-Criteria Analysis Methods for Fire Risk Assessment " [5], por plantear una estructura jerárquica aplicable a todos los artículos y centrarse en el estudio del riesgo de incendio de manera general: 
Tabla 2: Artículos empleados para la selección de atributos e identificación de subcategorías.

\begin{tabular}{|c|c|c|c|}
\hline Titulo & Año & Autores & Lugar de publicación \\
\hline $\begin{array}{l}\text { Application of the Model based on Fuzzy Consistent Matrix and AHP } \\
\text { in the Assessment of Fire Risk of Subway Tunnel }\end{array}$ & 2014 & $\begin{array}{l}\text { Jun-peng Gao } \\
\text { Zhi-sheng Xu } \\
\text { Ding-li Liu } \\
\text { Huan-huan Cao }\end{array}$ & Changsha, China \\
\hline $\begin{array}{l}\text { Assessment on Logistics Warehouse fire Risk based on Analytic Hier- } \\
\text { archy Process }\end{array}$ & 2012 & REN Shaoyun & Langfang, Hebei, China \\
\hline Fire Risk Evaluation of Subway & 2009 & HUANG Jing & Lang fang, China \\
\hline $\begin{array}{l}\text { Fire risk assessment with scoring system, using the support vector ma- } \\
\text { chine approach }\end{array}$ & 2015 & $\begin{array}{l}\text { Chun Kit Lau } \\
\text { Kin Keung Lai } \\
\text { Yan Pui Lee } \\
\text { Jiangze Du }\end{array}$ & Hong Kong, China \\
\hline $\begin{array}{l}\text { Research on Standardization Method of Risk Assessment for Fire Pub- } \\
\text { lic Liability Insurance in Assembly Occupancies and Underwriting Au- } \\
\text { diting }\end{array}$ & 2011 & HAN Hai-yun & Lang fang, China \\
\hline Applying Multi-Criteria Analysis Methods for Fire Risk Assessment & 2015 & $\begin{array}{l}\text { Julia Pushkina } \\
\text { Vladimirs Jansons } \\
\text { Konstantins Didenko }\end{array}$ & Riga, Letonia \\
\hline $\begin{array}{l}\text { Fire risk assessment for large-scale commercial buildings based on } \\
\text { structure entropy weight method }\end{array}$ & 2017 & $\begin{array}{l}\text { Fang Liu } \\
\text { Shengzhong Zhao } \\
\text { Miaocheng Weng } \\
\text { Yongqiang Liu }\end{array}$ & Chongqing, China \\
\hline
\end{tabular}


- Las medidas de tratamiento del riesgo

- Las características propias de la industria

- La gestión para la atención de incendios.

En cada una de las categorías identificadas se agruparon las siguientes subcategorías que fueron identificadas en todos los artículos estudiados:

Tabla 3: Subcategorías en medidas de tratamiento del riesgo

\begin{tabular}{|l|}
\hline \multicolumn{1}{|c|}{ Medidas de tratamiento del riesgo } \\
\hline Fuentes de combustible \\
\hline Fuentes de ignición \\
\hline Dimensiones del edificio \\
\hline Resistencia al fuego de la estructura \\
\hline Diseño estructural \\
\hline Personas expuestas \\
\hline Departamento de bomberos \\
\hline Fuente de ignición externas \\
\hline
\end{tabular}

Tabla 4: Subcategorías en características propias de la industria

\begin{tabular}{|l|}
\hline \multicolumn{1}{|c|}{ Características propias de la industria } \\
\hline Extinción automática \\
\hline Detección, notificación y alarma. \\
\hline Instalaciones para la evacuación \\
\hline Compartimentación \\
\hline Control de humo \\
\hline Brigada de emergencias \\
\hline Extinción Manual \\
\hline Fuente de agua externa \\
\hline
\end{tabular}

Tabla 5: Subcategorías en gestión para la atención de incendios

\begin{tabular}{|l|}
\hline \multicolumn{1}{|c|}{ Gestión para la atención de incendios } \\
\hline Pruebas y mantenimiento \\
\hline Procedimientos y entrenamiento \\
\hline Planes de emergencia \\
\hline
\end{tabular}

\subsection{Nivel de importancia de las categorías.}

Para calcular el nivel de importancia de cada una de las categorías se seleccionaron tres artículos que presentan una estructura de agrupación de las subcategorías similar y calcular el nivel de importancia de las categorías y se decide utilizar el promedio entre cada categoría de los artículos como el nivel de importancia de la categoría para este estudio apoyándose en la sugerencia presentada por algunos autores [4]. 
Tabla 6: Nivel de importancia de categorías del artículo "Fire risk assessment with scoring system, using the support vector machine approach" [7]

\begin{tabular}{|c|c|}
\hline \multicolumn{2}{|c|}{$\begin{array}{c}\begin{array}{c}\text { Fire risk assessment for large-scale commercial buildings based on } \\
\text { structure entropy weight method }\end{array}\end{array}$} \\
\hline $\begin{array}{ll}\text { Categoría } \\
\end{array}$ & Peso \\
\hline Factores que causan incendios & 0.2209 \\
\hline Métodos de protección pasiva & 0.1989 \\
\hline Métodos de protección activa & 0.2675 \\
\hline Gestión de atención de incendios & 0.1845 \\
\hline Habilidad de lucha contra incendios de la brigada & 0.1282 \\
\hline Total & 1 \\
\hline Categoría & Peso \\
\hline Las características propias de la industria & 0.2209 \\
\hline Las medidas de tratamiento del riesgo & 0.5946 \\
\hline La gestión para la atención de incendios & 0.1845 \\
\hline Total & 1 \\
\hline
\end{tabular}

Tabla 7: Nivel de importancia de categorías del artículo "Applying Multi-Criteria Analysis Methods for Fire Risk Assessment" [6]

\begin{tabular}{|c|c|}
\hline Applying Multi-Criteria Analysis Methods for Fire Risk A & sment \\
\hline Categoría & Peso \\
\hline $\begin{array}{l}\text { Existencia de medidas de prevención y protección de incen- } \\
\text { dios }\end{array}$ & 0.369 \\
\hline Características de la edificación & 0.462 \\
\hline Gestión para la atención de incendios & 0.169 \\
\hline Total & 1 \\
\hline Categoría & Peso \\
\hline Las características propias de la industria & 0.462 \\
\hline Las medidas de tratamiento del riesgo & 0.369 \\
\hline La gestión para la atención de incendios & 0.169 \\
\hline Total & 1 \\
\hline
\end{tabular}

Tabla 8: Nivel de importancia de categorías del artículo "Fire risk assessment for large-scale commercial buildings based on structure entropy weight method" [8]

\begin{tabular}{|c|c|c|}
\hline $\begin{array}{r}\text { Fire risk assessment with scoring system, u } \\
\text { approach }\end{array}$ & support ve & machine \\
\hline Categoría & Puntos & Peso \\
\hline Fuentes de Combustible & 144 & 0.072 \\
\hline Fuentes de ignición & 272 & 0.136 \\
\hline Identificación de personas en riesgo & 422 & 0.211 \\
\hline Rutas de evacuación & 292 & 0.146 \\
\hline Equipos de extinción y detección de incendios & 288 & 0.144 \\
\hline Procedimiento y entrenamiento & 170 & 0.085 \\
\hline Pruebas y mantenimiento & 272 & 0.136 \\
\hline Registro de seguridad & 140 & 0.07 \\
\hline Total & 2000 & 1 \\
\hline Categoría & Peso & \\
\hline Las características propias de la industria & 0.419 & \\
\hline Las medidas de tratamiento del riesgo & 0.29 & \\
\hline La gestión para la atención de incendios & 0.291 & \\
\hline Total & 1 & \\
\hline
\end{tabular}


Tabla 9: Nivel de importancia entre las categorías.

\begin{tabular}{l|r}
\hline \multicolumn{1}{c}{ Promedio tres estudios } \\
\hline \multicolumn{1}{c}{ Categoría } & Peso \\
\hline Las características propias de la industria & 0.36730000 \\
Las medidas de tratamiento del riesgo & 0.41786667 \\
La gestión para la atención de incendios & 0.21483333 \\
\hline Total & $\mathbf{1}$ \\
\hline
\end{tabular}

\subsection{Nivel de importancia de las subcategorías.}

Para determinar el grado de importancia de cada subcategoría se conformó un panel de 18 expertos pertenecientes al sector del diseño de protecciones contra incendios, del sector asegurador y del sector Bomberos. Cada uno de los expertos aplicó el proceso de análisis jerárquico para realizar las comparaciones pareadas de las subcategorías dentro de cada categoría.

En las figuras 1, 2 y 3 se muestra el resultado del ordenamiento jerárquico de los atributos según los expertos para cada una de las categorías.

Ordenamiento jerárquico de las subcategorias para gestión para la atención de incendios.

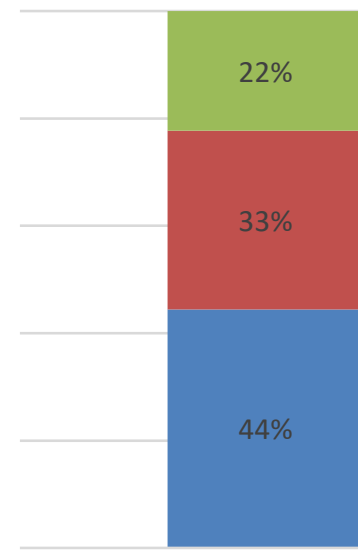

$1^{\circ}$

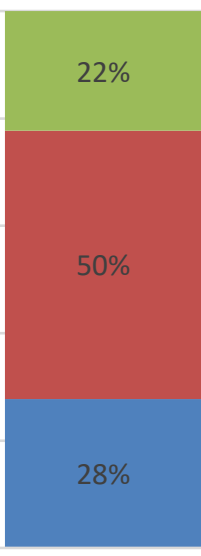

$2^{\circ}$

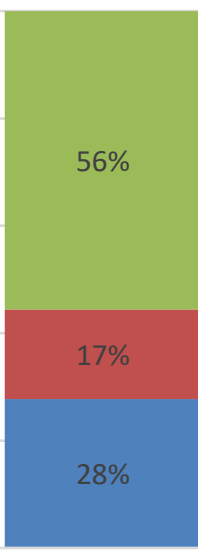

$3^{\circ}$

- Pruebas y mantenimiento

- Procedimientos y entrenamiento

— Planes de emergencia

Figura 1: Ordenamiento jerárquico según los expertos para gestión para la atención de incendio. 
Ordenamiento jerárquico de las subcategorias para características de la industria

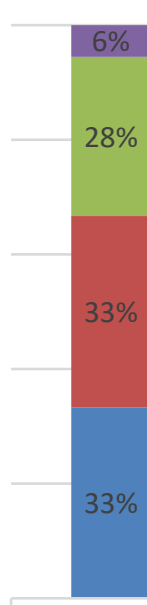

$1^{\circ}$

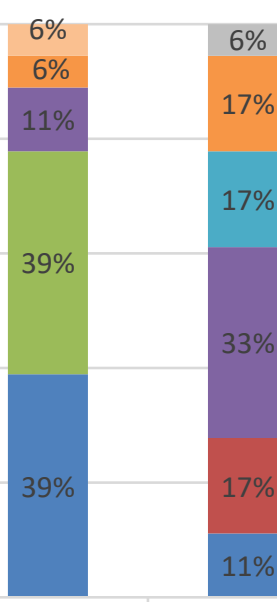

$2^{\circ}$

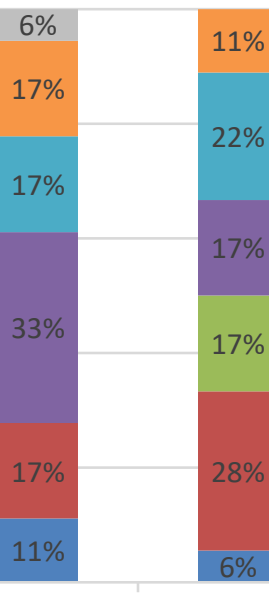

$3^{\circ}$

$4^{\circ}$

- Fuentes de combustible

- Fuentes de Ignición

- Diseño estructural

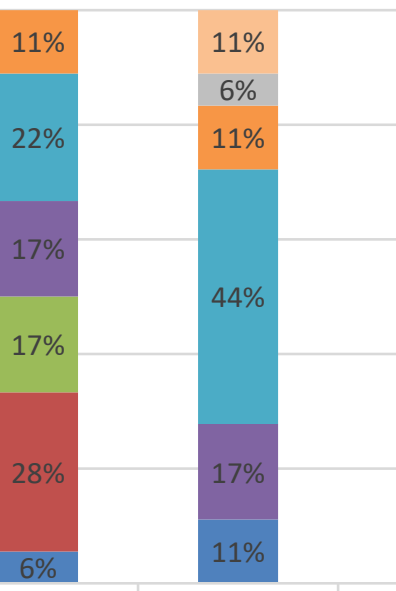

$5^{\circ}$

- Personas expuestas

- Resistencia al fuego de la estructura

- Dimensiones de la edificación

Figura 2: Ordenamiento jerárquico según los expertos para características de la industria.

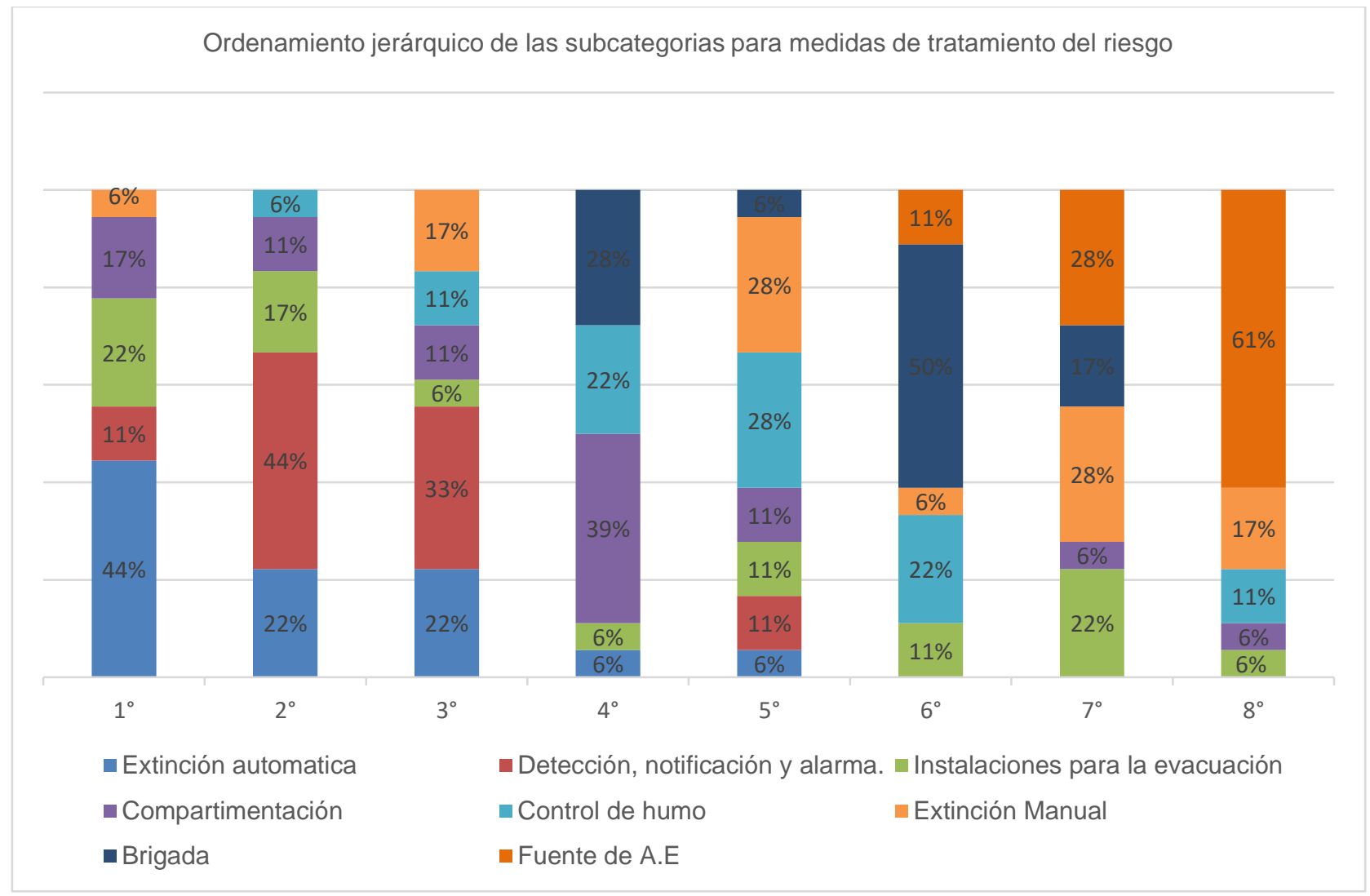

Figura 3: Ordenamiento jerárquico según los expertos para medidas de tratamiento del riesgo. 
DOI: http://dx.doi.org/ 10.33571/rpolitec.v17n33a5 La matriz de comparaciones pareadas para cada una de las categorías se construyó partiendo del consenso existente entre los expertos y para aquellas posiciones donde no se logró consenso entre los expertos se usó la consistencia. Para este trabajo al igual que lo hizo Larsson [6] se utilizó el promedio como el grado definitivo en la comparación pareada cuando el primer cuartil no era menor que en un grado y el tercer cuartil no era mayor que en un grado al ser comparados con el valor de la mediana.

Tabla 10: Cálculo de prioridades para Características de la industria.

\begin{tabular}{|c|c|c|c|c|c|c|c|c|c|}
\hline 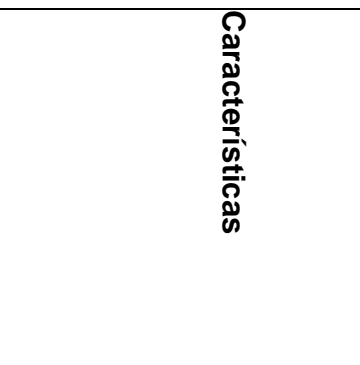 & 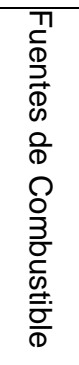 & 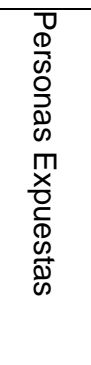 & 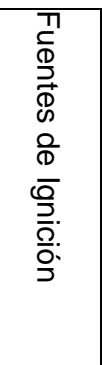 & 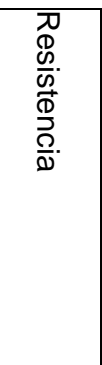 & 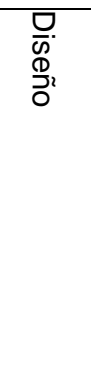 & 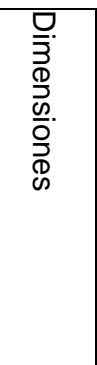 & 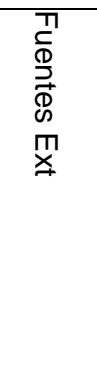 & 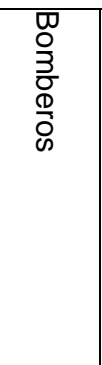 & 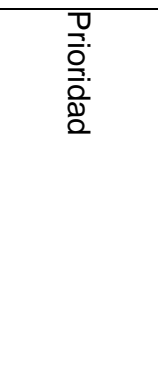 \\
\hline Fuentes de Combustible & 0.35 & 0.541 & 0.365 & 0.257 & 0.243 & 0.244 & 0.17 & 0.152 & 0.290144 \\
\hline Personas Expuestas & 0.12 & 0.18 & 0.365 & 0.257 & 0.243 & 0.244 & 0.17 & 0.152 & 0.2160674 \\
\hline Fuentes de Ignición & 0.12 & 0.06 & 0.122 & 0.257 & 0.243 & 0.244 & 0.17 & 0.152 & 0.1705806 \\
\hline Resistencia & 0.12 & 0.06 & 0.041 & 0.086 & 0.081 & 0.147 & 0.17 & 0.152 & 0.1065547 \\
\hline Diseño & 0.12 & 0.06 & 0.041 & 0.086 & 0.081 & 0.049 & 0.17 & 0.152 & 0.0943454 \\
\hline Dimensiones & 0.07 & 0.036 & 0.024 & 0.029 & 0.081 & 0.049 & 0.102 & 0.152 & 0.0678539 \\
\hline Fuentes Ext & 0.07 & 0.036 & 0.024 & 0.017 & 0.016 & 0.016 & 0.034 & 0.065 & 0.0348626 \\
\hline Bomberos & 0.05 & 0.026 & 0.017 & 0.012 & 0.012 & 0.007 & 0.011 & 0.022 & 0.0195915 \\
\hline
\end{tabular}

Tabla 11: Cálculo de prioridades para medidas de tratamiento del riesgo.

\begin{tabular}{|c|c|c|c|c|c|c|c|c|c|}
\hline 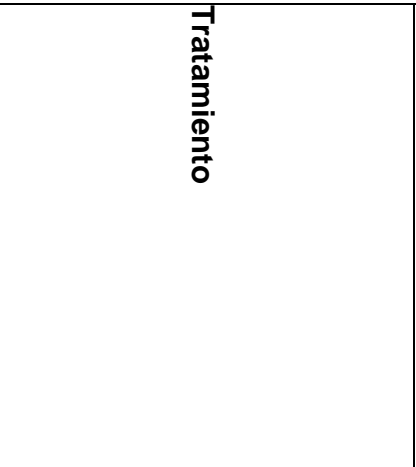 & 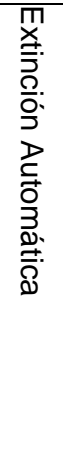 & 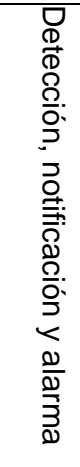 & 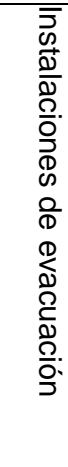 & 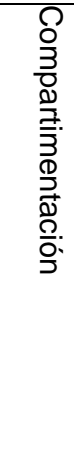 & 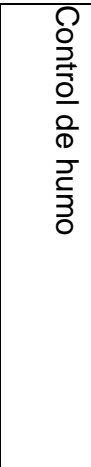 & 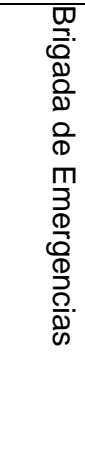 & 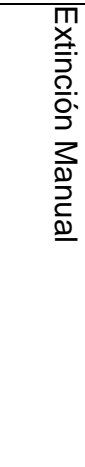 & 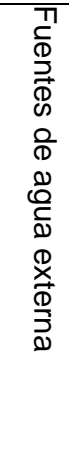 & $\begin{array}{l}\text { ㄲ. } \\
\text { 음. } \\
\text { ㅇ. } \\
\stackrel{0}{0}\end{array}$ \\
\hline Extinción Automática & 1 & 3 & 3 & 3 & 5 & 7 & 7 & 7 & 0.3452 \\
\hline $\begin{array}{l}\text { Detección, notificación y } \\
\text { alarma }\end{array}$ & 0.3 & 1 & 3 & 3 & 3 & 3 & 3 & 7 & 0.2022 \\
\hline Instalaciones de evacuación & 0.3 & 0.3 & 1 & 1 & 1 & 3 & 3 & 5 & 0.1098 \\
\hline Compartimentación & 0.3 & 0.3 & 1 & 1 & 1 & 3 & 3 & 5 & 0.1098 \\
\hline Control de humo & 0.2 & 0.3 & 1 & 1 & 1 & 3 & 3 & 5 & 0.1035 \\
\hline Brigada de Emergencias & 0.1 & 0.3 & 0.3 & 0.3 & 0.3 & 1 & 1 & 5 & 0.0533 \\
\hline Extinción Manual & 0.1 & 0.3 & 0.3 & 0.3 & 0.3 & 1 & 1 & 5 & 0.0533 \\
\hline Fuentes de agua externa & 0.1 & 0.1 & 0.2 & 0.2 & 0.2 & 0.2 & 0.2 & 1 & 0.022 \\
\hline
\end{tabular}


Tabla 12: Cálculo de prioridades para Gestión para la atención de incendios.

\begin{tabular}{|c|c|c|c|c|}
\hline 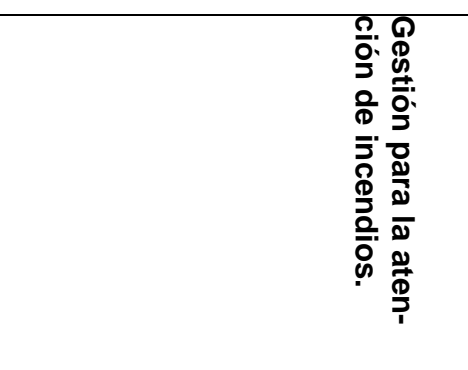 & 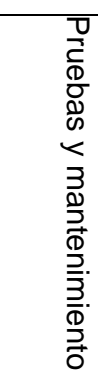 & 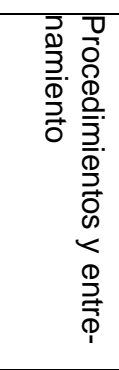 & 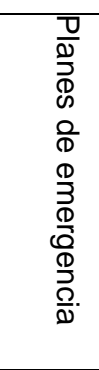 & 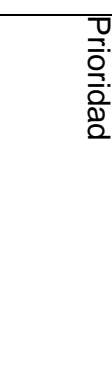 \\
\hline Pruebas y mantenimiento & 1 & 1 & 3 & 0.4285 \\
\hline Procedimientos y entrenamiento & 1 & 1 & 3 & 0.4285 \\
\hline Planes de emergencia & 0.33 & 0.33 & 1 & 0.1428 \\
\hline
\end{tabular}

Para validar el nivel de coherencia conseguido en las matrices de comparación para cada una de las categorías se realizó el cálculo de consistencia y se validó que su valor fuera inferior al $10 \%$, un valor adecuado cuando el número de factores comparados es inferior a 10 [7].

Tabla 1: Consistencia CR para Características de la industria.

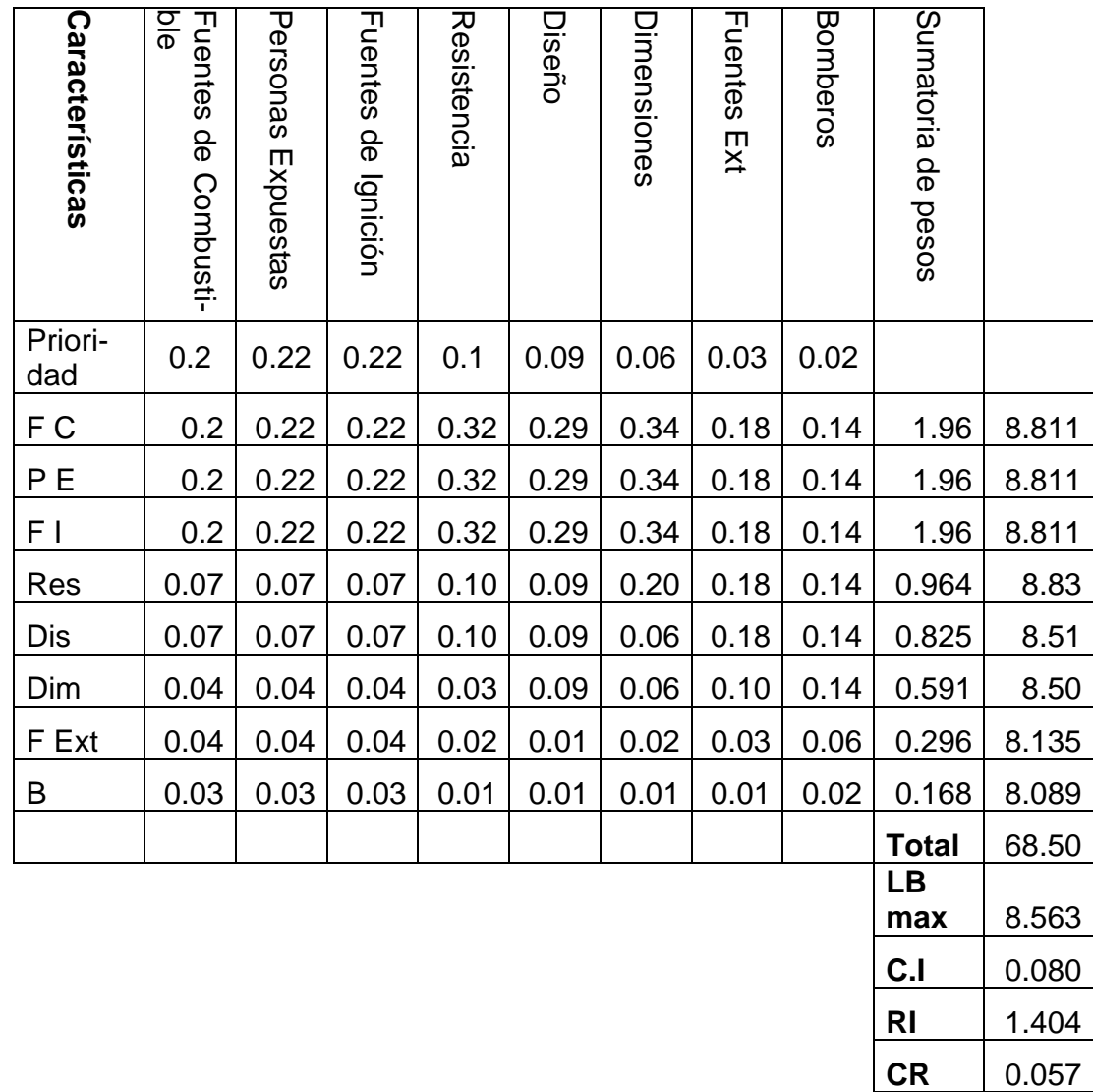


Tabla 15: Consistencia CR para gestión para la atención de incendios.

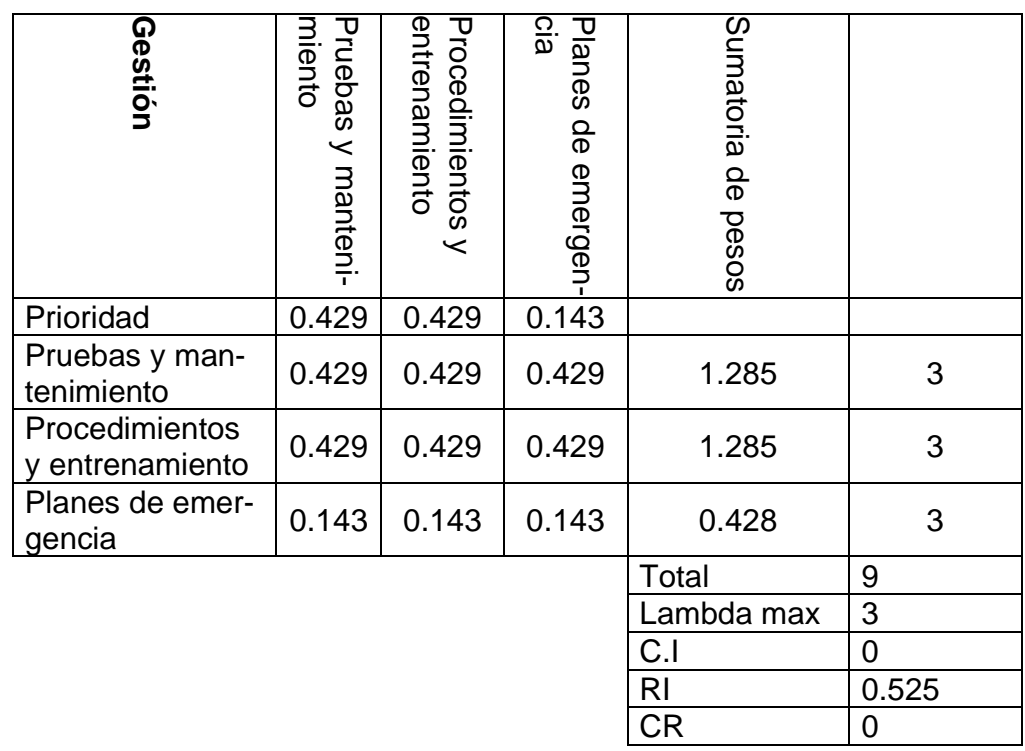

\section{CONCLUSIONES}

- Los atributos que caracterizan el riesgo de incendio y su nivel de importancia permitirán a los gestores de riesgos enfocar sus esfuerzos en aquellas condiciones que tienen una mayor relevancia sobre el nivel de riesgo.

- En el AHP de las características de la industria, se presentó una división entre los expertos que consideraban que los factores asociados a las fuentes de combustible y de ignición eran los más relevantes y entre los expertos que consideraban que las personas expuestas era el factor más relevante. Esta situación se puede ver explicada en la muy conocida practica de decir que el propósito principal de la gestión del riesgo de incendio es proteger la vida.

- La gestión del riesgo de incendio a través de las medidas de tratamiento del riesgo y la gestión para la atención de las emergencias tiene una importancia preponderante en la gestión del riesgo de incendio con un peso de $63,26 \%$.

- Las medidas de tratamiento del riesgo tienen el mayor peso dentro de la gestión del riesgo de incendio en las industrias, esta situación significa para los gestores de riesgos de las industrias considerar las protecciones automáticas y el diseño seguro si quiere obtener una reducción importante en la exposición al riesgo de incendio.

- Los sistemas automáticos de extinción de incendios y los sistemas automáticos de detección de incendios son los atributos más relevantes dentro de las medidas de tratamiento del riesgo, esta situación refleja la necesidad de gestionar el riesgo de incendio por medio de estas medidas.

- El desarrollo de los planes de emergencia para la atención de incendios en industrias requiere la disponibilidad de las medidas de tratamiento del riesgo como sistemas automáticos de extinción, a los sistemas automáticos de detección notificación y alarma, a los sistemas manuales de extinción de incendios, a la existencia de medios de evacuación para tener una reducción significativa en el nivel de riesgo. 


\section{REFERENCIAS}

[1] W. Meade. (1991). A First Pass at Computing the Cost of Fire Safety in a Modern society. Chapel Hill, NC: The Herndom Group, Inc, pp. I-2.

[2] D. Rasbash, G. Ramachandran, B. Kandola, J. Watts y M. Law. (2009). Evaluation of fire safety. Chichester: John Wiley \& Sons Ltd.

[3] T. L. Saaty. (2008). Decision making with the analytic hierarchy process. Int. J. Services Sciences, Vol. 1, No. 1,, pp. 83-98.

[4] R. L. Keeny y Raiffa, H. (1976). Decisions with multiple objectives. New York: John Wiley.

[5] J. Pushkina, J. Vladimirs y D. Konstantins. (2015). Applying Multi-Criteria Analysis Methods for Fire Risk Assessment. DE GRUYTER; Safety of Technogenic Environment.

[6] D. Larsson. (2000). Developing the Structure of a Fire Risk Index Method for Timber-frame Multi-storey Apartment Buildings. Department of Fire Safety Engineering, Lund University, Sweden.

[7] T. L. Saaty. (2012) Decision Making for Leaders: The Analytic Hierarchy Process for Decisions in a Complex World., Pittsburgh: RWS Publications.

[8] J. M. Moreno Jiménez, «EL PROCESO ANALíTICO JERÁRQUICO (AHP). FUNDAMENTOS, METODOLOGÍA Y APLICACIONES.,» Dpto. Métodos Estadísticos. Facultad de Económicas, Zaragoza..

[9] C. KitLau, K. Keung Lai, Y. Pui Lee y J. Du. (2015). Fire risk assessment with scoring system, using the support vector machine approach. Fire Safety Journal, p. 188-195.

[10] F. Liu, S. Zhao, M. Weng y Y. Liu. (2017). Fire risk assessment for large-scale commercial buildings based on structure entropy weight method. Safety Science, p. 26-40. 